\title{
Black soldier fly and gut health in broiler chickens: insights into the relationship between cecal microbiota and intestinal mucin composition
}

Ilaria Biasato", Ilario Ferrocino ${ }^{1}$, Sihem Dabbou², Rocchina Evangelista², Francesco Gai ${ }^{3}$, Laura Gasco ${ }^{1 *}$ (D, Luca Cocolin ${ }^{1}$, Maria Teresa Capucchio ${ }^{2,3}$ and Achille Schiavone $e^{2,3}$

\begin{abstract}
Background: The relationship between diet and intestinal microbiota and mucin composition appears to be fundamental for poultry gut health. The effects of insect meal (whose role as alternative feed ingredient is now well recognized) on gut microbiota and mucin composition have recently been reported in Tenebrio molitor-fed free-range and broiler chickens, but no data are currently available for Hermetia illucens (HI)-fed broilers. The present study evaluated the effects of dietary $\mathrm{HI}$ meal inclusion on cecal microbiota and intestinal mucin composition of broiler chickens.

Results: A total of 256 male broiler chickens were allotted to 4 dietary treatments (control diet [C] and 5\%, 10\% and $15 \% \mathrm{HI}$ meal inclusion, with 8 replicate pens/treatment and 8 birds/pen) and slaughtered at $35 \mathrm{~d}$ of age (2 animals/pen, 16 birds/diet). The cecal microbiota assessment by $16 \mathrm{~S}$ rRNA amplicon based sequencing showed lower alpha diversity in HI15 chickens (Shannon, $P<0.05$ ) and higher beta diversity (Adonis and ANOSIM, $P<0.001$ ) in birds fed $\mathrm{HI}$ diets than C. Furthermore, HI15 birds displayed significant increase of the relative abundance of Proteobacteria phylum (False Discovery Rate [FDR] < 0.05) when compared to HI10. L-Ruminococcus (Ruminococcus from Lachnospiraceae family), Faecalibacterium, Blautia and Clostridium genera were found to be characteristic of HI5 cecal microbiota (FDR < 0.05), while broiler chickens fed HI10 and HI15 diets were characterized $(F D R<0.05)$ by Lactobacillus and Ruminococcus (HI10) and Bacteroides, Roseburia and Helicobacter genera (HI15). Periodic-acid Schiff, Alcian Blue pH 2.5 and high iron diamine staining on small and large intestine also demonstrated lower mucin staining intensity in the intestinal villi of HI10 and HI15 birds than $\mathrm{C}(P<0.05)$.

Conclusions: Dietary HI meal utilization at low inclusion levels (i.e., 5\%) positively influenced either the cecal microbiota or the gut mucin dynamics in terms of selection of potentially beneficial bacteria and increase in villi mucins. However, high inclusion levels (in particular the 15\%) may have a negative influence in terms of partial reduction of microbial complexity, reduction of potentially beneficial bacteria, selection of bacteria with mucolytic activity and decrease in villi mucins.
\end{abstract}

Keywords: $16 \mathrm{~S}$ rRNA, Gut health, Hermetia illucens L., Histochemistry, Insect meal, Microbiota, Mucin, Poultry

\footnotetext{
* Correspondence: laura.gasco@unito.it

1 Department of Agricultural, Forest and Food Sciences, University of Turin,

Largo Paolo Braccini 2, 10095 Grugliasco, TO, Italy

Full list of author information is available at the end of the article
}

(c) The Author(s). 2020 Open Access This article is distributed under the terms of the Creative Commons Attribution 4.0 International License (http://creativecommons.org/licenses/by/4.0/), which permits unrestricted use, distribution, and reproduction in any medium, provided you give appropriate credit to the original author(s) and the source, provide a link to the Creative Commons license, and indicate if changes were made. The Creative Commons Public Domain Dedication waiver (http://creativecommons.org/publicdomain/zero/1.0/) applies to the data made available in this article, unless otherwise stated. 


\section{Background}

Optimal gastrointestinal health and functionality is essential for sustainable animal production, since it has direct repercussions on both the animal health and the performance [1]. The gut barrier (comprising the microbiota and their products, mucus layers, host-derived antimicrobial compounds, epithelium, and underlying immune tissue) constantly interacts with the dietary nutrients, in order to maintain the delicate balance needed for preventing the passage of harmful microorganisms and substances into the animal body [2]. In particular, the relationship between the diet and the gut microbiota and mucin dynamics appears to be fundamental. As a first aspect to consider, one of the main defense components of the gastrointestinal environment against the enteric pathogens is represented by the intestinal microbiota. Indeed, imbalances of gut microbiota-host interaction are frequently associated with several intestinal disorders [3]. In parallel, the mucus layers represent the first host-derived line of defense in the intestine [4]. Mucus, which is mainly composed of mucins, traps the pathogenic bacteria and promotes their expulsion from the intestine via the luminal flow, as well as having a lubricant activity, modulates the digestion and absorption of the nutrients, and provides the colonization sites and nutrients for the commensal microbes [5, 6]. Bacterial colonization and proliferation has been reported to have a key role in determining the mucin composition, both by the synthesis of mucin-specific glycosidases, glycosulfatases and proteases $[7,8]$ and the modulation of mucin gene expression [9]. There is also evidence that several feed substances may widely affect the complex, delicate relationship existing between the gut microbiota and mucin dynamics in poultry, either by directly modifying the intestinal mucin composition [10, 11], or indirectly by modulating the intestinal microbial population [12, 13].

Insects as novel, alternative feed ingredients has now become a worldwide, well-recognized research topic in animal nutrition, because of their excellent nutritive properties and peculiar rearing characteristics [14, 15]. Indeed, insects contain high quality and quantity of protein [14] and they can easily be reared on several organic side streams, thus reducing their environmental and economic impact and allowing their transformation into high-protein feeds [15]. In particular, yellow mealworm (Tenebrio molitor, TM) and black soldier fly (Hermetia illucens, HI) larvae are characterized by a remarkable nutritional profile in terms of crude protein (CP: $52.8 \pm$ $4.2 \%[\mathrm{TM}]$ and $42.1 \pm 1.0 \%[\mathrm{HI}]$ ) and ether extract (EE: $36.1 \pm 4.1[\mathrm{TM}]$ and $26.0 \pm 8.3[\mathrm{HI}])$ contents that make them extremely promising for poultry feeding [14]. Dietary TM meal inclusion has recently been reported to significantly influence the gut health of free-range [16] and broiler [17] chickens, in particular by affecting both their cecal microbiota (in terms of modified phylum and genus profile) and their intestinal mucin dynamics (in terms of altered villi mucins). However, no data about the modulation of gut microbiota and mucin composition by $\mathrm{HI}$ meal utilization are currently available in poultry.

Based on these considerations, the present study investigates the effects of dietary HI larva meal inclusion on cecal microbiota and intestinal mucin composition of broiler chickens.

\section{Methods}

\section{Birds and experimental design}

The experimental design of the present study is described in details in the research published by Dabbou et al. [18]. Briefly, a total of 256 1-day-old male broiler chicks (Ross 708) were randomly distributed into four isonitrogenous and isoenergetic dietary treatments, each consisting of 8 pens as replicates $(1.0 \mathrm{~m}$ wide $\times 1.5 \mathrm{~m}$ long, equipped with a feeder, an automatic drinker and rice hulls as bedding) with 8 chicks per pen. The control diet $(C)$ was based on maize meal, corn gluten meal and soybean meal, while the experimental diets were obtained including $5 \%, 10 \%$ and $15 \%$ of a partially defatted HI larva meal (Hermetia Deutschland GmbH \& Co. KG, Baruth/Mark, Germany) as partial replacement of soybean meal, corn gluten meal and soybean oil (HI5, HI10 and HI15 groups, respectively). The chemical composition of the HI meal was as follows: $942 \mathrm{~g} / \mathrm{kg}$ of diet of dry matter (DM), $553 \mathrm{~g} / \mathrm{kg}$ DM of crude protein $(\mathrm{CP})$, $180 \mathrm{~g} / \mathrm{kg}$ DM of ether extract (EE) and $24.4 \mathrm{MJ} / \mathrm{kg} \mathrm{DM}$ of gross energy. Detailed information about the diets is summarized in Additional file 1. Nutrients digestibility, apparent metabolizable energy (AME) and apparent metabolizable energy corrected for nitrogen balance (AMEn) of the HI meal used for feed formulation were previously assessed [19]. All the birds were reared under the same environmental conditions (lighting schedule: $18 \mathrm{~h}$ light: $6 \mathrm{~h}$ dark; $\mathrm{T}: 32^{\circ} \mathrm{C}$ during the first day, with reduction by $4{ }^{\circ} \mathrm{C}$ per week according to the age of the broilers until it reached $20^{\circ} \mathrm{C}$ ) throughout the whole experimental trial and fed ad libitum. The chickens received regular vaccination against Newcastle disease, Marek disease, infectious bronchitis and coccidiosis at hatching. The growth performance of the broiler chickens were also evaluated throughout the experimental trial, as reported in details by Dabbou et al. [18]. Briefly, the live weight $(\mathrm{LW})$, the average daily gain (ADG), the average daily feed intake (DFI) and the feed conversion ratio (FCR) of the birds increased with increasing levels of dietary HI meal inclusion (LW: end of the starter, the grower and the finisher periods; ADG: starter and grower periods; DFI: starter period; FCR: grower and 
finisher periods, and overall). The experimental period lasted $35 \mathrm{~d}$.

\section{Intestinal sampling and processing}

A total of 16 chickens per treatment ( 2 birds/pen) were randomly selected and slaughtered in a commercial abattoir at the end of the experimental trial. Cecal content was sampled into sterile plastic tubes with a sterile spatula, immediately refrigerated (for a maximum of $2 \mathrm{~h}$ ) and frozen at $-80^{\circ} \mathrm{C}$ until DNA extraction. Welldefined, standardized segments of both the small (duodenum, jejunum and ileum) and the large (cecum) intestine were sampled and processed for histochemical staining, as previously reported by Biasato et al. [16].

\section{DNA extraction and sequencing}

A pool of the cecal content from 2 chickens per pen $(8$ pools per feeding group) was submitted to DNA extraction and sequencing. The DNA was extracted with the RNeasy Power Microbiome KIT (Qiagen, Milan, Italy) following the instructions reported by the manufacturer. One $\mu \mathrm{L}$ of RNase (Illumina Inc., San Diego, CA) was added to digest the RNA in the DNA samples, with an incubation of $1 \mathrm{~h}$ at $37^{\circ} \mathrm{C}$. The DNA was quantified using the NanoDrop and standardized at $5 \mathrm{ng} / \mu \mathrm{L}$. Due to poor DNA quality, one samples belonging to the HI5 group was excluded. The extracted DNA was used to assess the microbiota by the amplification of the V3-V4 region of the 16S rRNA gene using the following primers: $16 \mathrm{~S}-\mathrm{F}$ ( $5^{\prime}$ - TCG TCG GCA GCG TCA GAT GTG TAT AAG AGA CAG CCT ACG GGN GGC WGC AG-3') and 16S-R (5'-GTC TCG TGG GCT CGG AGA TGT GTA TAA GAG ACA GGA CTA CHV GGG TAT CTA ATC C-3') [20]. Twenty-five $\mu \mathrm{L}$ PCR reactions were prepared using $12.5 \mu \mathrm{L}$ of the $2 \mathrm{X}$ KAPA HiFi HotStart Ready Mix Taq (Kapa Biosystems, Wilmington, MA), $1 \mu \mathrm{mol} / \mathrm{L}$ of each primer and $2.5 \mu \mathrm{L}$ of DNA. A total of 25 cycles of $30 \mathrm{~s}$ of denaturation $\left(95^{\circ} \mathrm{C}\right), 30 \mathrm{~s}$ of primer annealing $\left(55^{\circ} \mathrm{C}\right)$ and $30 \mathrm{~s}$ of primer elongation $\left(72^{\circ} \mathrm{C}\right)$, followed by a final elongation step $\left(72^{\circ} \mathrm{C}\right)$ of $5 \mathrm{~min}$, were carried out. The PCR products were purified by means of an Agencourt AMPure kit (Beckman Coulter, Milan, Italy) and the resulting products were tagged by using the Nextera XT Index Kit (Illumina Inc., San Diego, CA) according to the guidelines reported by the manufacturer. Sequencing was performed with a MiSeq Illumina instrument (Illumina) with V3 chemistry and generated $250 \mathrm{bp}$ paired-end reads according to the manufacturer's instructions. The software used for the base-calling and Illumina barcode demultiplexing processes were the MiSeq Control Soft. V2.3.0.3, the RTA v1.18.42.0 and the CASAVA v1.8.2.

\section{Histochemical staining}

The intestinal sections of 16 chickens per dietary treatment (2 birds/pen) were submitted to three different histochemical staining, as previously reported by Biasato et al. [16]: periodic-acid Schiff (for the identification of the neutral mucins), Alcian Blue $\mathrm{pH} 2.5$ (for the identification of the acidic sialylated mucins) and high iron diamine (for the identification of the acidic sulfated mucins).

\section{Mucin staining intensity}

The mucin staining intensity of the goblet cells was assessed on one slide per histochemical staining for each intestinal segment by using a well-defined, semiquantitative score, according to Biasato et al. [16].

\section{Bioinformatics and statistical analysis}

Paired-end reads were merged by FLASH software [21] with default parameters. QIIME 1.9.0 was used for quality filtered (at Phred < Q20) [22] and the recently described pipeline [23] was adopted. The Operational Taxonomic Units (OTUs) clustering was performed at 97\% of similarity [24] and centroids sequence were used to assign taxonomy by the Greengenes $16 \mathrm{~S}$ rRNA gene database (version 2013). Alpha diversity indices were calculated using the diversity function of the vegan package [25]. Diet-related differences were assessed by pairwise t-test, Kruskal-Wallis tests or Wilcoxon rank sum test as appropriate. $P$ values were adjusted for multiple testing and a false discovery rate $(\mathrm{FDR})<0.05$ was considered statistically significant. Weighted UniFrac distance matrices were used to perform Adonis and ANOSIM statistical tests in the R environment (https:// www.r-project.org). A filtered OTU table was generated at $0.1 \%$ abundance in at least 2 samples through QIIME. The so-obtained table was used to determine the Principal component analysis (PCA) in the $\mathrm{R}$ environment. The OTU table displayed the highest taxonomy resolution reached by the $16 \mathrm{~S}$ data. Indeed, when the genus level was not reached by the taxonomy assignment, the bacterial family, order or phyla were actually showed.

The statistical analysis of the histochemical data was performed using IBM SPSS Statistics V20.0.0 software. The histochemical scores were analyzed using the generalized linear model (GLM) recently adopted by Biasato et al. [16]. Results were expressed as least squares means and SEM. $P$ values $<0.05$ were considered statistically significant.

\section{Results}

\section{Cecal microbiota characterization}

A total of $1,716,304$ raw reads $(2 \times 250 \mathrm{bp})$ were obtained after sequencing. After joint and quality filtering, a total of 1,602,517 reads passed the filters applied through 
QIIME, with an average value of 69,674 reads/sample (SD: 21,342) and a median sequence length of $441 \mathrm{bp}$. In order to avoid potential biases due to different sequencing depths, all the samples were rarefied at 3600 reads after the raw read quality filtering. The rarefaction analysis and the Good's coverage indicated a satisfactory coverage for all the samples (average Good's coverage of $94 \%$ ).

Dietary HI meal inclusion significantly affected the diversity within the microbial populations, as indicated by a lower Shannon index $(P<0.05)$ observed in the broiler chickens fed HI15 (6.49) compared to the other diets $(\mathrm{C}=7.25$; HI5 $=6.88$; HII0 = 7.36). However, the other alpha diversity measures showed no significant differences $(P>0.05)$ among the $C$ (average PD Whole Tree: 79.29; average Chao1: 2680.28; average observed species richness: 1161.33), HI5 (average PD Whole Tree: 73.93; average Chao1: 2418.11; average observed species richness: 1024.80), HI10 (average PD Whole Tree: 77.42; average Chao1: 2318.36; average observed species richness: 1085.50) and HI15 groups (average PD Whole Tree: 69.23; average Chao1: 2160.36; average observed species richness: 956.33). Adonis and ANOSIM statistical tests based on Weighted UniFrac distance matrix showed significant differences between the $\mathrm{C}$ and the $\mathrm{HI}$ groups in the relative abundance of the microbial species $(P<0.001)$. Indeed, the PCA showed a clear shift of the cecal microbiota as a function of the dietary HI meal inclusion (Fig. 1).

Relative abundances of the main phyla and genera in the broiler chickens of the present study obtained by 16S rRNA gene sequencing are summarized in Fig. 2 and Additional file 2. Firmicutes represented the dominant phylum of the cecal microbiota in either the $\mathrm{C}$ or the HI groups, outnumbering the Proteobacteria and Bacteoidetes phyla (Fig. 2a, Additional file 2). Within the phylum Firmicutes, Unclassified members (U. m.) of Clostridiales order, U. m. of Ruminococcaceae family, Faecalibacterium, Oscillospira, U. m. of Lachnospiraceae family, Ruminococcus, L-Ruminococcus (Ruminococcus belonging to Lachnospiraceae family), U. m. of Erysipelotrichaceae family and Lactobacillus were identified as the main OTUs in the birds fed both the $\mathrm{C}$ and the HI diets (Fig. 2b). Helicobacter was the dominant member of the Proteobacteria phylum in either the $C$ or the HI groups (Fig. 2b). Within the phylum Bacteroidetes, Bacteroides was observed as predominant OTU in the animals fed both the $\mathrm{C}$ and the $\mathrm{HI}$ diets (Fig. 2b, Additional file 2).

Compared to the $\mathrm{C}$ group (Fig. 3), the birds fed HI displayed unaffected relative abundances of Firmicutes and Bacteroidetes phyla (FDR > 0.05). On the contrary, the relative abundance of Proteobacteria was higher in the HI15 animals than the HI10 (FDR $<0.05)$. The birds fed

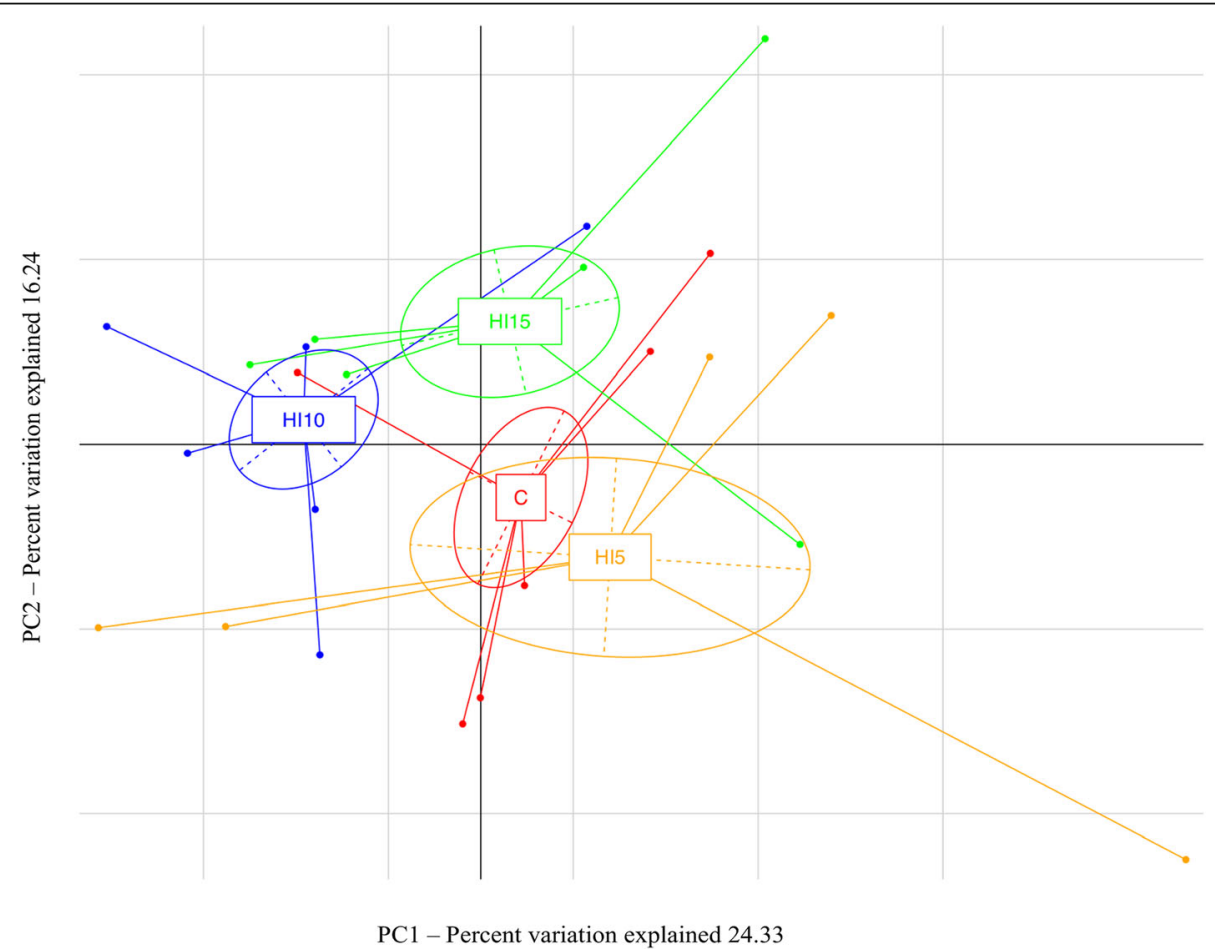

Fig. 1 Bacterial community composition (weighted UniFrac beta diversity, PCA plots) in cecal samples of broiler chickens fed control (C), 5\% (HI5), $10 \%(\mathrm{HI} 10)$ and 15\% (HI15) inclusion level of Hermetia illucens meal diets 

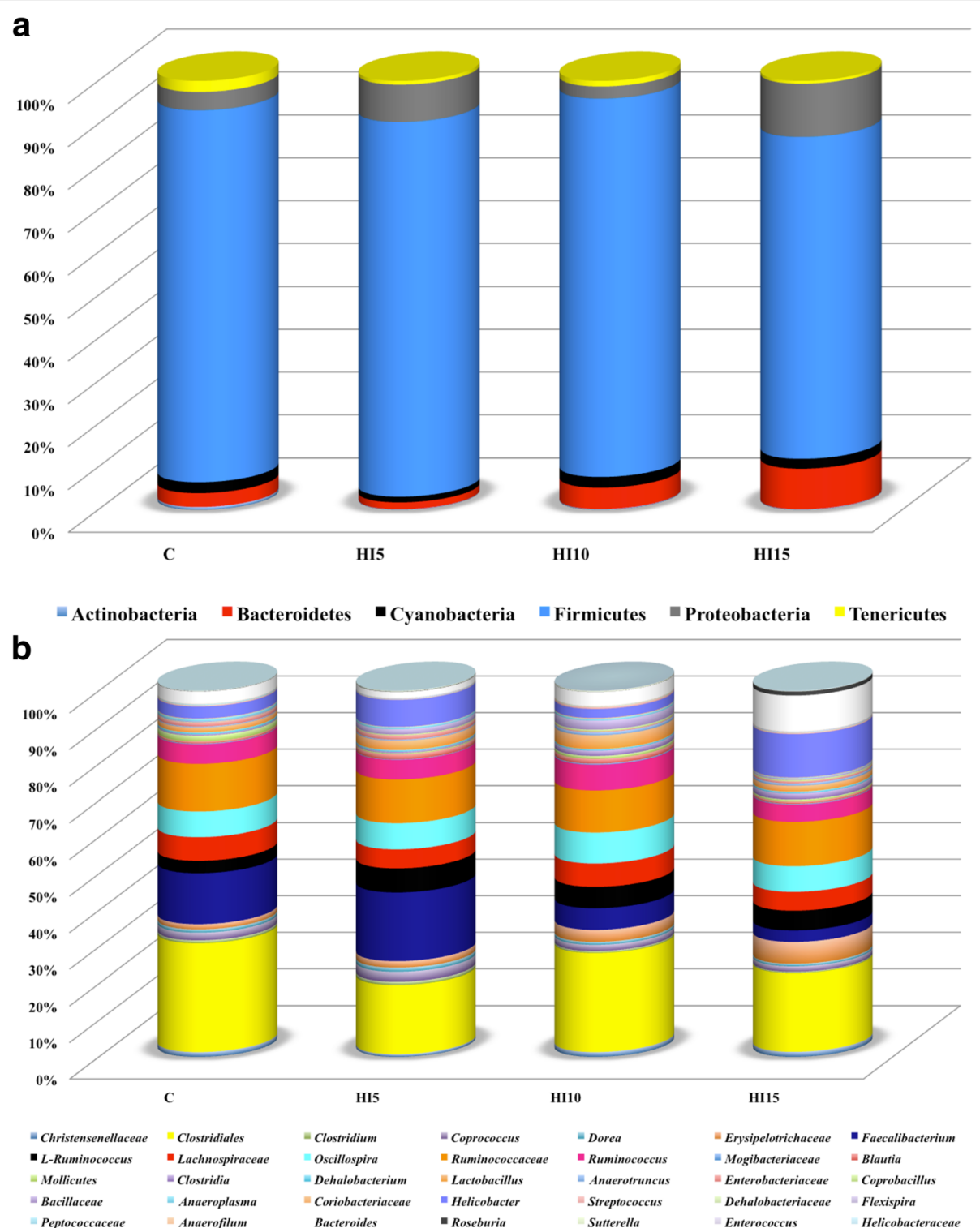

Fig. 2 Relative abundance of the main bacterial phyla (a) and genera (b) in cecal samples of broiler chickens fed control (C), 5\% (HI5), 10\% (HI10) and $15 \%(H \mid 15)$ inclusion level of Hermetia illucens meal diets

HI also showed unaffected Firmicutes:Bacteroidetes ratios compared to the $\mathrm{C}$ group (FDR $<0.05)$. Comparing the relative abundances of the main OTUs among the dietary treatments, a specific microbiota signature was observed for each diet. In particular, the broiler chickens fed $\mathrm{C}$ were characterized by the presence of $\mathrm{U}$. m. of Lachnospiraceae family $(\mathrm{FDR}<0.05)$, while L-Ruminococcus (Ruminococcus from Lachnospiraceae family), Faecalibacterium, Blautia and Clostridium genera were found to be characteristic of the HI5 diet (FDR $<0.05$ ). The broiler chickens fed HI10 were characterized (FDR $<0.05)$ by Lactobacillus and Ruminococcus OTUs, whereas Bacteroides, Roseburia and Helicobacter genera were characteristic for the HI15 diet (FDR < 0.05).

\section{Intestinal mucin composition}

The mucin type $(P<0.001)$, the gut segment $(P<$ $0.001)$ and the crypt fragment $(P<0.001)$ significantly influenced the mucin staining intensity in the intestinal crypts, while the histochemical findings were unaffected by dietary HI meal inclusion $(P>0.05$, Table 1). In particular, the crypts showed higher neutral and acidic sialylated mucins staining intensity $(P<0.001)$ than the acidic sulfated (Fig. 4). Higher mucin staining intensity was also found in the ileal crypts $(P<0.001)$ when compared to the other gut segments. Furthermore, the crypt base showed greater mucin staining intensity $(P<0.001)$ than the midsection and tip, with a significant decrease $(P<0.001)$ 


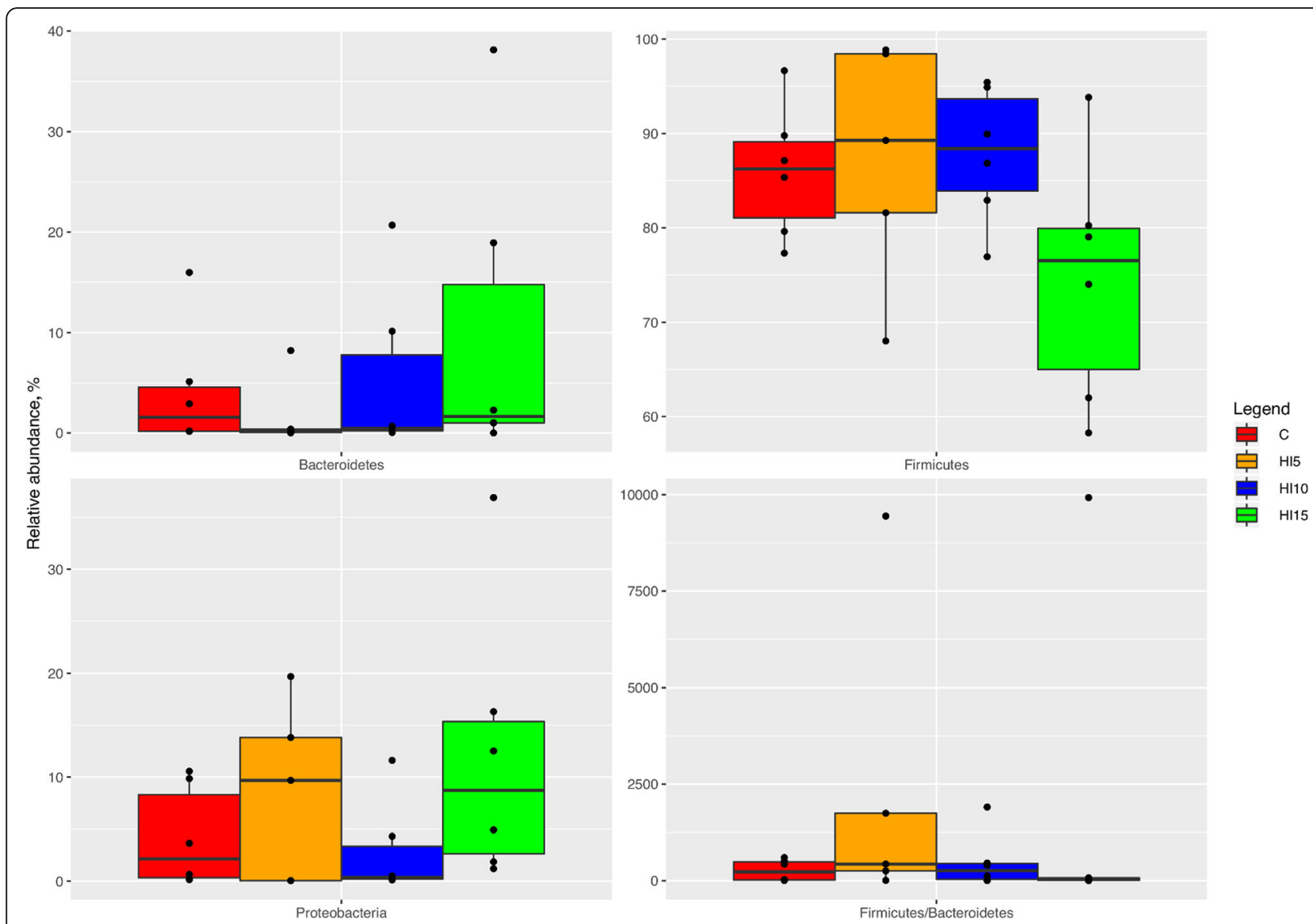

Fig. 3 Relative abundance at phylum level of differentially abundant OTUs in cecal samples of broiler chickens fed control (C), 5\% (HI5), 10\% (HI10) and 15\% (HI15) inclusion level of Hermetia illucens meal diets. Pairwise Kruskal-Wallis test, FDR $<0.05$

Table 1 Effects of diet, mucin type, gut segment and cryptvillus fragment on mucin staining intensity in the broiler chickens

\begin{tabular}{llll}
\hline Factor & d.f. $^{f}$ & Chi-square & $P^{*}$ \\
\hline Crypts $^{\text {Diet }}{ }^{\mathrm{a}}$ & 3 & & \\
Mucin type $^{\mathrm{b}}$ & 2 & 3.751 & 0.290 \\
Gut segment $^{\mathrm{c}}$ & 3 & 22.566 & $<0.001$ \\
Fragment $^{\mathrm{d}}$ & 2 & 247.461 & $<0.001$ \\
Villi $_{\text {Diet }}$ & & & $<0.001$ \\
Mucin type & 2 & 25.497 & $<0.001$ \\
Gut segment & & 4.510 & 0.100 \\
Fragment & 2 & 571.512 & $<0.001$ \\
\hline
\end{tabular}

${ }^{\mathrm{a}}$ Four dietary treatments: $\mathrm{C}=$ control; $\mathrm{HI} 5=5 \%$ inclusion level of Hermetia illucens; $\mathrm{HI} 10=10 \%$ inclusion level of Hermetia illucens; $\mathrm{HI} 15=15 \%$ inclusion level of Hermetia illucens

${ }^{\text {b} T h r e e ~ t y p e s: ~ n e u t r a l, ~ a c i d i c ~ s i a l y l a t e d ~ a n d ~ a c i d i c ~ s u l f a t e d ~ m u c i n s ~}$

${ }^{c}$ Four gut segments: duodenum, jejunum, ileum and caecum

${ }^{\mathrm{d}}$ Three fragments: base, midsection and tip

'Three gut segments: duodenum, jejunum and ileum

fDegrees of freedom

*Statistical significance: $P<0.05$. Statistical trend: $P \leq 0.10$ being also observed from the midsection to the tip (Table 2).

The mucin staining intensity in the intestinal villi of the broiler chickens significantly depended on the dietary treatment $(P<0.001)$ and the gut segment $(P<$ 0.001 ), whereas there was no significant influence of both the mucin type and the villus fragment $(P>0.05)$ on the histochemical scores (Table 1). In particular, the villi of the HI10 and the HI15 animals showed lower mucin staining intensity $(P<0.01)$ than $\mathrm{C}$ and HI5. Greater acidic sialylated mucin staining intensity $(P<$ 0.05 ) than the acidic sulfated was also observed (Fig. 5). Furthermore, the ileal villi showed higher mucin staining intensity $(P<0.001)$ than the other gut segments, with a significant increase $(P<0.001)$ being also identified from the duodenum to the jejunum (Table 2).

\section{Discussion}

In the current research, the attention was focused on the cecal microbiota as chicken ceca harbor the highest microbial cell densities and diversity, have the longest residence time of digesta in the gastrointestinal tract, and 


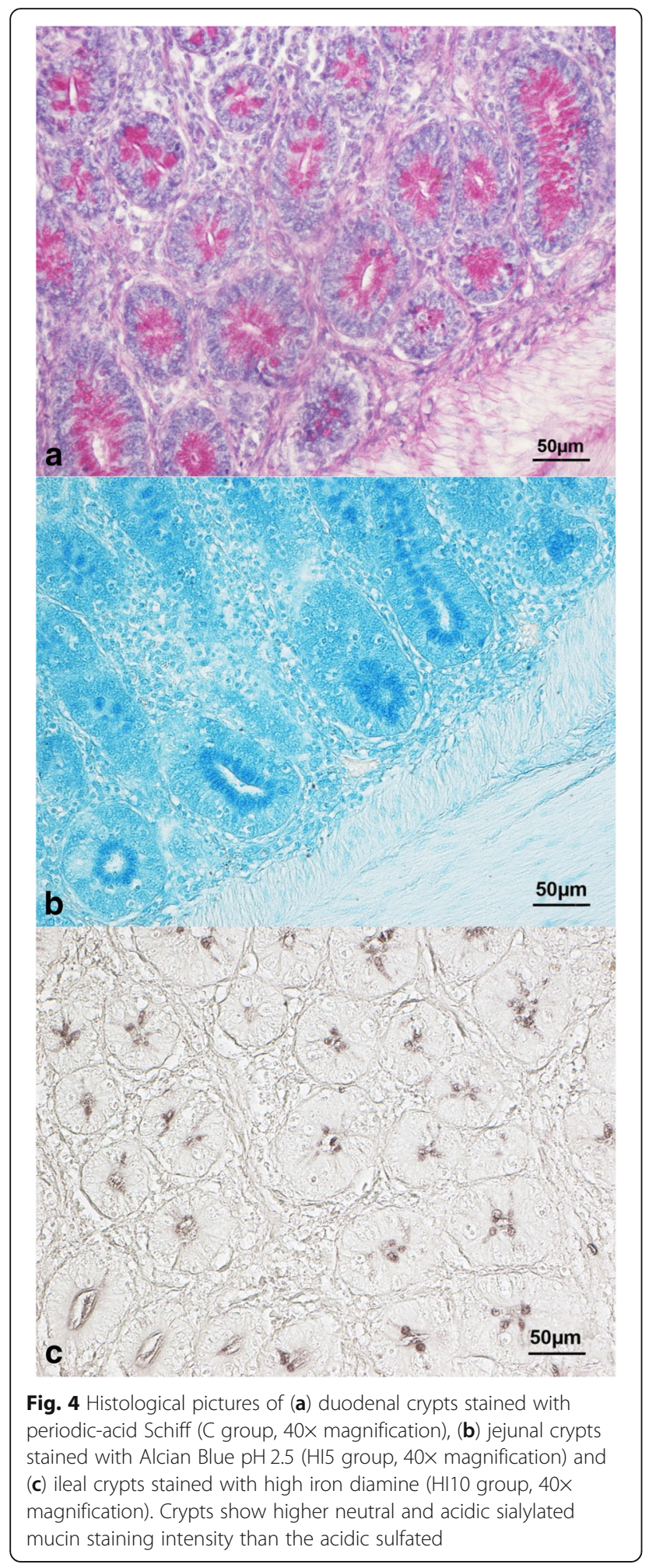

are an important site for recycling of urea, water regulation, and carbohydrate fermentations contributing to intestinal health and nutrition [26].

Firmicutes, Proteobacteria and Bacteroidetes represented the most abundant bacterial phyla detected in the
Table 2 Least square means of mucin staining intensity in the intestinal crypts of the broiler chickens in relation to diet, mucin type, gut segment and crypt fragment

\begin{tabular}{|c|c|c|c|}
\hline & Factor & Factor levels & $\begin{array}{l}\text { Mucin staining } \\
\text { intensity }{ }^{1,2}\end{array}$ \\
\hline \multirow[t]{14}{*}{ Crypts } & \multirow[t]{4}{*}{ Diet } & C & $1.23 \pm 0.03$ \\
\hline & & HI5 & $1.27 \pm 0.03$ \\
\hline & & HI10 & $1.27 \pm 0.03$ \\
\hline & & HI15 & $1.30 \pm 0.03$ \\
\hline & \multirow[t]{3}{*}{ Mucin type } & Neutral & $1.32 \pm 0.03^{\mathrm{A}}$ \\
\hline & & Acidic sialylated & $1.31 \pm 0.02^{\mathrm{A}}$ \\
\hline & & Acidic sulfated & $1.18 \pm 0.02^{\mathrm{B}}$ \\
\hline & \multirow[t]{4}{*}{ Gut segment } & Duodenum & $1.26 \pm 0.03^{B}$ \\
\hline & & Jejunum & $1.20 \pm 0.03^{B C}$ \\
\hline & & Ileum & $1.45 \pm 0.03^{\mathrm{A}}$ \\
\hline & & Caecum & $1.19 \pm 0.03^{C}$ \\
\hline & \multirow[t]{3}{*}{ Fragment } & Base & $1.59 \pm 0.03^{\mathrm{A}}$ \\
\hline & & Midsection & $1.17 \pm 0.02^{B}$ \\
\hline & & Tip & $1.10 \pm 0.02^{c}$ \\
\hline \multirow[t]{13}{*}{ Villi } & \multirow[t]{4}{*}{ Diet } & $C$ & $1.90 \pm 0.04^{\mathrm{A}}$ \\
\hline & & HI5 & $1.87 \pm 0.04^{\mathrm{A}}$ \\
\hline & & H10 & $1.72 \pm 0.04^{B}$ \\
\hline & & HI15 & $1.66 \pm 0.04^{B}$ \\
\hline & \multirow[t]{3}{*}{ Mucin type } & Neutral & $1.79 \pm 0.04^{\mathrm{ab}}$ \\
\hline & & Acidic sialylated & $1.84 \pm 0.04^{\mathrm{a}}$ \\
\hline & & Acidic sulfated & $1.74 \pm 0.03^{b}$ \\
\hline & \multirow[t]{3}{*}{ Gut segment } & Duodenum & $1.23 \pm 0.03^{C}$ \\
\hline & & Jejunum & $1.95 \pm 0.04^{B}$ \\
\hline & & Ileum & $2.37 \pm 0.04^{\mathrm{A}}$ \\
\hline & \multirow[t]{3}{*}{ Fragment } & Base & $1.75 \pm 0.03$ \\
\hline & & Midsection & $1.81 \pm 0.04$ \\
\hline & & Tip & $1.80 \pm 0.04$ \\
\hline
\end{tabular}

C control, HI5 5\% inclusion level of Hermetia illucens, HI10 10\% inclusion level of Hermetia illucens, HI15 15\% inclusion level of Hermetia illucens

${ }^{1}$ Data are represented as mean of counts \pm SEM

${ }^{2}$ Means with different superscript letters $(a, b$ or $A, B, C)$ within the same column per predictor (i.e. diet, mucin type, gut segment or fragment) differ significantly $(P<0.05$ or $P<0.01$, respectively)

cecal microbiota of both the $\mathrm{C}$ - and the HI-fed broiler chickens of the present study. The predominance of Firmicutes over Bacteroidetes is in agreement with the previous researches [26-29], while the predominance of Proteobacteria over Bacteroidetes clearly contrasts and appears difficult to explain. The Proteobacteria phylum comprises many pathogenic bacteria, such as Escherichia coli, Salmonella spp., Vibrio cholera and Helicobacter spp. Furthermore, in human patients high numbers of Proteobacteria members are usually indicative of a bad intestinal health and have a crucial role in the development of some gastrointestinal health conditions such as 


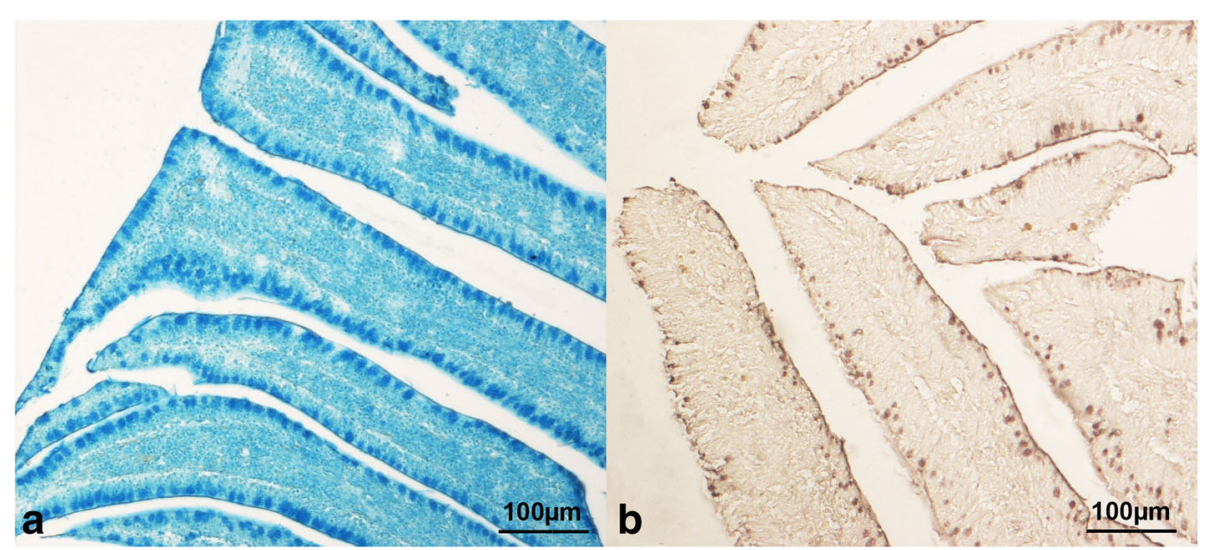

Fig. 5 Histological pictures of ileal villi stained with (a) Alcian Blue pH 2.5 (HI15 group, 10x magnification) and (b) high iron diamine (C group, 10x magnification). Villi show higher acidic sialylated mucin staining intensity than the acidic sulfated

the chronic dysbiosis [30] and the inflammatory bowel disease [31]. However, an important aspect that must always be taken into consideration is that several host(i.e., age, sex and breed) and environmental-related factors (i.e., biosecurity level, housing, litter, feed access and climate) may widely influence the chicken intestinal microbiota [32], thus potentially explaining the differences between the current and the previous researches. As a partial confirmation of this aspect, Biasato et al. [17] observed an unexpected predominance of the phylum Bacteroidetes over Firmicutes in female broiler chickens fed both the $\mathrm{C}$ - and the TM-based diets.

The cecal microbiota of the birds fed either the $C$ or the HI-based diets in the present study was mainly colonized by Clostridiales order, members of Ruminococcaceae, Faecalibacterium and Oscillospira genera, and Lachnospiraceae family. These findings reflect the currently available literature, where the main bacterial genera identified in the chicken cecum have been reported to be Clostridium, Ruminococcus, Lactobacillus, Bacteroides [28, 33-36] and, to a lesser extent, Alistipes and Faecalibacterium [28]. The identification of a physiological cecal community also confirms what was observed in a previous research about dietary TM meal inclusion in diets for broilers [17].

Investigating the differences in the 16S rRNA gene sequences between the $\mathrm{C}$ - and the HI-fed broiler chickens of the current research, the first aspect to consider is that birds fed the $15 \%$ level of $\mathrm{HI}$ meal inclusion showed lower Shannon index when compared to the other diets. Differently, higher $\beta$-diversity was observed in the broiler chickens fed HI-based diets than $\mathrm{C}$ (with a particularly evident distinction between the broilers fed the $5 \%$ level of HI meal inclusion and the other diets), as already reported for insect-fed laying hens [37], free-range poultry [16] and broiler chickens [17]. High levels of bacterial diversity have been associated with a maintained stability of the intestinal microbiota after environmental stress factors [38], as well as an effective colonization resistance against the potential pathogenic bacteria [39]. Based on these considerations, insect meal utilization (especially at low inclusion levels) may be advantageous for modulating the complexity of the chicken intestinal microbiota.

Despite no significant differences being observed in regards to phyla composition between the birds fed the $\mathrm{C}$ and the HI-based diets in the present study, a specific signature at genus level was, however, detected in their cecal microbiota. In particular, bacteria capable of producing several end products that may affect the intestinal health were identified [40].

In regards to the broiler chickens fed the $\mathrm{C}$ diet, Lachnospiraceae family was observed as characteristic OTU of their cecal microbiota. It is well known that Lachnospiraceae, along with Ruminococcaceae, is a typical butyrate-producing family [41]. Butyrate has various positive properties, since it represents an important nutrition source for the enterocytes, stimulates the gut mucin production [42] and improves tight-junction integrity [43]. It is also involved in the cellular differentiation and proliferation within the intestinal mucosa [44] and is capable of reducing the inflammatory response as an anti-inflammatory effector [45].

Secondly, the birds fed the $5 \%$ level of HI meal inclusion showed L-Ruminococcus (Ruminococcus belonging to Lachnospiraceae family), Faecalibacterium, Blautia and Clostridium as characteristic OTUs of their cecal microbiota. Analogously to the already described members of Lachnospiraceae family, Faecalibacterium genus encompasses members capable of producing butyric acid [46]. Furthermore, Blautia is a new genus belonging to the Ruminococcaceae family that can produce short chain fatty acids (SCFAs) through the glucose metabolism and digest cellulose in food [47]. The SCFAs 
production is fundamental for the optimal intestinal health, since they represent a remarkable source of energy for enterocytes [48] and have the ability to suppress the enteric pathogens [49]. Last but not least, Clostridium is one of the main bacterial genera detected in the chicken cecum [28, 33, 34], being also capable of producing butyrate [50]. Increased abundance of Clostridium genus has also been reported in TM-fed broiler chickens [17].

In regards to the broiler chickens fed the $10 \%$ level of HI meal inclusion, Lactobacillus and Ruminococcus were identified as characteristic OTUs of their cecal microbiota. These bacterial genera are frequently identified in the normal chicken microbiota [28, 33-36]. However, the most relevant finding is that Lactobacillus positively stimulates the homeostasis of immune cells and the host gut health [51, 52]. The lactate produced by Lactobacillus species can also be converted to SCFAs [53-55], whose positive properties have already been described. Interestingly, TM meal utilization has previously been reported to reduce the relative abundance of Ruminococcus genus in the chicken cecal microbiota [17], thus suggesting a potential different way of action of the two insect types.

As a final aspect to consider, the birds fed the 15\% level of HI meal inclusion showed Bacteroides, Roseburia and Helicobacter as characteristic OTUs of their cecal microbiota. Apart from being one of the most predominant members of chicken microbiota [28, 33-36], Bacteroides genus may significantly contribute to the gut health of the animals. Its positive effects are related to its beneficial role for weight gain and growth performance [56] and the inhibition of Clostridium perfringens sporulation by its fermentation products [57]. Furthermore, Roseburia is a well-known butyrate-producing genus [58], thus further representing another potential beneficial bacterium. However, a potential negative finding may be represented by the remarkable identification of Helicobacter genus. Indeed, some specific enterohepatic Helicobacter species (i.e., Helicobacter pullorum) have been detected in gut and liver of hens with vibrionic-like liver lesions and humans with gastroenteritis [59]. Furthermore, bacteria such as Helicobacter pylori possess the enzymatic ability to disrupt the oligomeric structure of mucin and are capable of downregulating the mucin synthesis [8]. Interestingly, the animals fed the $10 \%$ and $15 \%$ levels of $\mathrm{HI}$ meal inclusion (especially the 15\%) showed reduced mucin production in the intestinal villi, thus suggesting a direct interaction between the microbiota and the mucin dynamics. This also confirms what was recently reported in broilers chickens diets containing the $10 \%$ inclusion level of TM meal, which displayed decrease in villi mucins and high abundance of Helicobacter genus [17].
In the current research, higher mucin staining intensity was observed in the intestinal villi of the broiler chickens fed the $5 \%$ level of $\mathrm{HI}$ meal inclusion when compared to the $10 \%$ and $15 \%$, with the latter also showing lower mucin staining intensity than the $\mathrm{C}$ group. Forder et al. [60] pointed out that the microbial flora may influence the mucin production, since some bacteria (i.e., Helicobacter pylori) are known to possess a strong mucolytic activity [7] that induces the chicken gut to increase the sialomucin production as defense strategy [60]. As already mentioned before, the birds fed the $15 \%$ level of HI meal inclusion displayed Helicobacter genus as one of the characteristic OTUs of their cecal microbiota. Therefore, a direct relationship between the reduced intestinal mucin production and the identified bacterial population appears to be reasonable. Mucins also constitute a digestion- and absorption-assisting medium and protect the gut environment against the pathogenic bacteria [5]. Therefore, independently of the intestinal microbiota changes, the utilization of $\mathrm{HI}$ meal at low inclusion rates (i.e., 5\%) may be preferable to preserve the protective properties of the mucin glycoproteins in order to optimize the digestive process and to prevent the enteric infections, as already suggested by Biasato et al. [17].

Independently of HI meal utilization, the intestinal crypts of the broiler chickens in the present study showed lower acidic sulfated mucin staining intensity than other mucin types. Greater acidic sialylated mucin staining intensity than acidic sulfated was also observed in the intestinal villi. Despite the limited information currently available about the mucin dynamics in crypts and villi, the physiological relevance of the different mucin subtypes has, however, been suggested. In particular, the production of neutral mucins has been recognized as a protective mechanism against the enteric pathogens [61] and as a promoter of the intestinal maturity for facilitating the complex carbohydrate breakdown [60]. Sialic acid groups have also several protective properties [62] and increase in acidic sialylated mucins production has been hypothesized to represent a defense strategy against mucus degradation by bacterial colonization [60]. Finally, a high degree of sulfation within the acidic mucins is also characteristic for immature goblet cells [63]. Therefore, the assessment of mucin types in the current research is indicative of overall mature and healthy guts with a well-developed mucin secretory architecture.

Independently of insect meal inclusion, both the intestinal crypts and the villi of the birds in the present study showed higher mucin staining intensity in the ileum compared with the other gut segments. This is in agreement with the previous findings in chickens, which revealed an increased density of the goblet cells from the 
duodenum to the ileum $[16,17,60,64]$. Since the distal ileum has been suggested as a preferred region for bacterial colonization, the above-mentioned mucin dynamics may reflect the need for great protection and subsequent high mucin synthesis [60].

The intestinal crypts of the broiler chickens fed both the $\mathrm{C}$ and the HI-based diets in the current research showed greater mucin staining intensity in the base compared with the other crypt fragments. A decreased stain in the tip represents the physiological condition in the intestinal crypts, as previously reported $[10,16,17$, $65]$. On the contrary, the intestinal villi showed unaffected mucin staining among the villus fragments. This finding disagrees with what was reported by Tsirtsikos et al. $[10,11]$, which found greater staining intensity at the villus tip and explained this scenario as a consequence of the key role of mucins in the gut epithelium $[10,11]$. However, the goblet cell proliferation may also occur along the entire length of the villus, thus potentially explaining the absence of differences among the villus fragments [65].

As final considerations, the changes in the cecal microbiota and the mucin dynamics observed in the present study may be attributed to both direct and indirect effects of $\mathrm{HI}$ meal. The positive increase in SCFAs-producing bacteria predominantly identified in the HI5- and HI10-fed birds could be related to their capability of directly degrading the chitin contained in the insect meal, as already suggested by Borrelli et al. [37]. On the contrary, the proliferation of potential mucolytic pathogens (with the subsequent reduction of villi mucins) observed in the HI15-fed broilers may indirectly be attributed to the increased dietary content of chitin, which can negatively affect the protein digestibility (as already suggested by Dabbou et al. [18]). Indeed, the increase in nondigested protein at ileal level can lead to hindgut protein fermentation, with formation of toxic compounds potentially capable of creating a non-healthy gut environment. Since the CP digestibility of the HI meal used in the current trial was also moderate (0.62) [19], this explanation seems reasonable. Another aspect to recall in relation to the gut mucosal characteristics of the birds in the present study is that the broiler chickens fed the 15\% level of HI meal inclusion also showed the worst gut morphology in terms of short villi, deep crypts and reduced villus height to crypt depth ratios. Furthermore, the same birds displayed worse growth performance than the other chickens in terms of higher feed conversion ratios [18]. Since the rapid growth of chickens directly depends on the morphological and the functional characteristics of the digestive tract [66], the relationship between the negative gut microbiota, morphology and mucin composition findings and the deterioration of the growth performance observed in the
HI15 birds of the current research seems logical, as already suggested by Biasato et al. [16].

\section{Conclusions}

In conclusion, dietary HI meal inclusion was demonstrated to modulate both the cecal microbiota and the gut mucin composition of the broiler chickens. In particular, insect meal utilization at low inclusion levels (i.e., 5\%) positively influenced either the cecal microbiota or the intestinal mucin dynamics in terms of preservation of physiological microbial populations, selection of potentially beneficial bacteria and increase in villi mucins. However, high inclusion levels (in particular the 15\%) may have a negative influence in terms of partial reduction of the microbial complexity, reduction of potentially beneficial bacteria, selection of bacteria with mucolytic activity and decrease in villi mucins. In particular, changes in butyrate- and SCFAs-producing bacteria seemed to have a crucial role, but further studies also adopting metatranscriptomic and meta-metabolomic approaches are mandatory to better contextualize these findings. Despite the observed potential negative modulation, the detection of physiological cecal community and intestinal mucin dynamics in all the animals (observed independently of insect meal utilization) represents a positive result in terms of gut health preservation and further stimulates the use of insects in poultry feeding.

\section{Supplementary information}

Supplementary information accompanies this paper at https://doi.org/10. 1186/s40104-019-0413-y.

\begin{abstract}
Additional file 1. Ingredients and chemical composition of the experimental diets. Mineral-vitamin premix: vitamin A (retinyl acetate), 12,500 IU; vitamin $D_{3}$ (cholecalciferol), $3000 \mathrm{IU}$; vitamin E (DL-a-tocopheryl acetate), $60 \mathrm{IU}$; vitamin $\mathrm{K}$ (menadione sodium bisulfite), $1.02 \mathrm{mg}$; riboflavin, $2.0 \mathrm{mg}$; pantothenate, $8.0 \mathrm{mg}$; niacin, $6 \mathrm{mg}$; piridossin, $4 \mathrm{mg}$; folic acid, 0.5 mg; biotin, 0.10 mg; tiamin, 1.0 mg; vitamin $B_{12}, 20$ mg; Mn, 120 mg; Zn, $80 \mathrm{mg}$; Fe, $52 \mathrm{mg}$; Cu, $15 \mathrm{mg} ; \mathrm{I}, 1.5 \mathrm{mg}$; Se, $0.4 \mathrm{mg}$. AME = apparent metabolizable energy; $\mathrm{CP}=$ crude protein; $\mathrm{EE}=$ ether extract; $\mathrm{CF}=$ crude fiber; $C=$ control; $\mathrm{HI} 5=5 \%$ inclusion level of Hermetia illucens; HI10 = 10\% inclusion level of Hermetia illucens; HI15 = 15\% inclusion level of Hermetia illucens.
\end{abstract}

Additional file 2. Relative abundance of the main bacterial phyla and genera of cecal microbiota of broiler chickens fed with control (C), 5\% (HI5), 10\% (HI10) and 15\% (HI15) inclusion level of Hermetia illucens meal diets.

\section{Abbreviations}

AME: Apparent metabolizable energy; AMEn: Apparent metabolizable energy corrected for nitrogen balance; CP: Crude protein; DM: Dry matter; EE: Ether extract; FDR: False discovery rate; HI: Hermetia illucens; OTU: Operational taxonomic unit; PCA: Principal component analysis; SCFAs: Short chain fatty acids; SEM: Standard error of the mean; TM: Tenebrio molitor; Vh: Villus height; Vh/Cd: Villus height to crypt depth ratio; WG: Weight gain

\section{Acknowledgments}

The authors gratefully acknowledge Mr. Dario Sola and Mr. Mario Colombano for technical support and Hermetia Deutschland GmbH \& Co. KG for the provision of the insect meal. 


\section{Authors' contributions}

$I B, L G$ and $A S$ conceived and designed the experiment. IB, IF, SD, FG, LC, MTC, LG and AS collected the experiments data. IB and MTC performed the histochemical investigations. If performed the $16 \mathrm{~S}$ rRNA amplicon based sequencing. RE processed the gut samples. IB and IF performed the statistical analysis. All authors interpreted the data. IB and IF wrote the first draft of the manuscript. All authors critically reviewed the manuscript for intellectual content and gave final approval for the version to be published.

\section{Funding}

Financial support for this work was provided by University of Turin (ex 60\%) grant (Es. fin. 2015-2016-2017).

\section{Availability of data and materials}

The datasets analysed in the present study are available from the corresponding author on reasonable request.

\section{Ethics approval and consent to participate}

The experimental protocol was designed according to the guidelines of the current European Directive (2010/63/EU) on the care and protection of animals used for scientific purposes and approved by the Ethical Committee of the Department of Veterinary Sciences of the University of Turin (Italy) (Ref. 1, 28/06/2016).

\section{Consent for publication}

Not applicable.

\section{Competing interests}

The authors declare that they have no competing interests.

\section{Author details}

'Department of Agricultural, Forest and Food Sciences, University of Turin, Largo Paolo Braccini 2, 10095 Grugliasco, TO, Italy. ${ }^{2}$ Department of Veterinary Sciences, University of Turin, Largo Paolo Braccini 2, 10095 Grugliasco, TO, Italy. ${ }^{3}$ Institute of Science of Food Production, National Research Council, Largo Paolo Braccini 2, 10095 Grugliasco, TO, Italy.

\section{Received: 23 July 2019 Accepted: 9 December 2019}

\section{Published online: 03 February 2020}

\section{References}

1. Kogut MH, Arsenault RJ. Editorial: gut health: the new paradigm in food animal production. Front Vet Sci. 2016;3:71.

2. Broom LJ. Gut barrier function: effects of (antibiotic) growth promoters on key barrier components and associations with growth performance. Poult Sci. 2018;97:1572-8. https://doi.org/10.3382/ps/pey021.

3. Pourabedin $M$, Zhao X. Prebiotics and gut microbiota in chickens. FEMS Microbiol Lett. 2015;362:fnv122. https://doi.org/10.1093/femsle/fnv122.

4. Forstner JF, Oliver MG, Sylvester FA. Production, structure and biologic relevance of gastrointestinal mucins. In: Guerrant RL, editor. Infections of the gastrointestinal tract. New York: Raven Press; 1995. p. 71-88.

5. Forstner G, Forstner JF. Gastrointestinal mucus. In: Johnson LR, editor. Physiology of the gastrointestinal tract. New York: Raven Press; 1994. p. 1255-84.

6. Zhang Q, Eicher SD, Applegate TJ. Development of intestinal mucin 2, IgA and polymeric Ig receptor expressions in broiler chickens and Pekin ducks. Poult Sci. 2015;94:172-80. https://doi.org/10.3382/ps/peu064.

7. Robertson AM, Wright DP. Bacterial glycosulphatases and sulphomucin degradation. Can J Gastroenterol. 1997;11:361-6. https://doi.org/10.1155/ 1997/642360.

8. Deplancke B, Gaskins HR. Microbial modulation of innate defense: goblet cells and the intestinal mucus layer. Am J Clin Nutr. 2001;73:1131S-41S. https://doi.org/10.1093/ajcn/73.6.1131S.

9. Mack DR, Michail S, Wei S, McDougall L, Hollingsworth MA. Probiotics inhibit enteropathogenic E. Coli adherence in vitro by inducing intestinal mucin gene expression. Am J Physiol Gastrointest Liver Physiol. 1999;276: G941-50. https://doi.org/10.1152/ajpgi.1999.276.4.G941.

10. Tsirtsikos P, Fegeros K, Kominakis A, Balaskas C, Mountzouris KC. Modulation of intestinal mucin composition and mucosal morphology by dietary phytogenic inclusion level in broilers. Animal. 2012a;6:1049-57. https://doi. org/10.1017/S1751731111002680.
11. Tsirtsikos P, Fegeros K, Balaskas C, Kominakis A, Mountzouris KC. Dietary probiotic inclusion level modulates intestinal mucin composition and mucosal morphology in broilers. Poult Sci. 2012b;91:1860-8. https://doi.org/ 10.3382/ps.2011-02005.

12. Pan D, Yu Z. Intestinal microbiome of poultry and its interaction with host and diet. Gut Microbes. 2014;5:108-19. https://doi.org/10.4161/gmic.26945.

13. Shang Y, Kumar S, Thippareddi H, Kim WK. Effect of dietary Fructooligosaccharide (FOS) supplementation on lleal microbiota in broiler chickens. Poult Sci. 2018;97:3622-34. https://doi.org/10.3382/ps/pey131.

14. Makkar HPS, Tran G, Heuzé V, Ankers P. State-of-the-art on use of insects as animal feed. Anim Feed Sci Technol. 2014;197:1-33.

15. Meneguz M, Schiavone A, Gai F, Dama A, Lussiana C, Renna M, et al. Effect of rearing substrate on growth performance, waste reduction efficiency and chemical composition of black soldier fly (Hermetia illucens) larvae. J Sci Food Agric. 2018;98:5776-84. https://doi.org/10.1002/jsfa.9127.

16. Biasato I, Ferrocino I, Biasibetti E, Grego E, Dabbou S, Sereno A, et al. Modulation of intestinal microbiota, morphology and mucin composition by dietary insect meal inclusion in free-range chickens. BMC Vet Res. 2018; 14:383. https://doi.org/10.1186/s12917-018-1690-y.

17. Biasato I, Ferrocino I, Grego E, Dabbou S, Gai F, Gasco L, et al. Gut microbiota and mucin composition in female broiler chickens fed diets including yellow mealworm (Tenebrio molitor, L.). Animals (Basel). 2019;9: E213. https://doi.org/10.3390/ani9050213.

18. Dabbou S, Gai F, Biasato I, Capucchio MT, Biasibetti E, Dezzutto D, et al. Black soldier fly defatted meal as a dietary protein source for broiler chickens: effects on growth performance, blood traits, gut morphology and histological features. J Anim Sci Biotechnol. 2018;9:49. https://doi.org/10. 1186/s40104-018-0266-9.

19. Schiavone A, De Marco M, Martínez S, Dabbou S, Renna M, Madrid J, et al. Nutritional value of a partially defatted and a highly defatted black soldier fly larvae (Hermetia illucens L.) meal for broiler chickens: apparent nutrient digestibility, apparent metabolizable energy and apparent ileal amino acid digestibility. J Anim Sci Biotechnol. 2017;8:51. https://doi.org/10.1186/ s40104-017-0181-5.

20. Klindworth A, Pruesse E, Schweer T, Peplies J, Quast C, Horn M, et al. Evaluation of general 165 ribosomal RNA gene PCR primers for classical and next-generation sequencing-based diversity studies. Nucleic Acids Res. 2013; 41:e1. https://doi.org/10.1093/nar/gks808.

21. Magoč T, Salzberg SL. FLASH: fast length adjustment of short reads to improve genome assemblies. Bioinformatics. 2011;27:2957-63. https://doi. org/10.1093/bioinformatics/btr507.

22. Caporaso JG, Kuczynski J, Stombaugh J, Bittinger K, Bushman FD, Costello EK, et al. QIIME allows analysis of high-throughput community sequencing data. Nat Methods. 2010;7:335-6. https://doi.org/10.1038/nmeth.f.303.

23. Ferrocino I, Bellio A, Romano A, Macori G, Rantsiou K, Decastelli L, et al. RNA-based amplicon sequencing reveals microbiota development during ripening of artisanal versus industrial lard d'arnad. Appl Environ Microbiol. 2017;83:e00983-17. https://doi.org/10.1128/AEM.00983-17.

24. Edgar RC. Search and clustering orders of magnitude faster than BLAST. Bioinformatics. 2010;26:2460-1. https://doi.org/10.1093/bioinformatics/btq461.

25. Dixon P. VEGAN, a package of $R$ functions for community ecology. J Veg Sci. 2003;14:927-30. https://doi.org/10.1111/j.1654-1103.2003.tb02228.x.

26. Wise MG, Siragusa GR. Quantitative analysis of the intestinal bacterial community in one- to three-week-old commercially reared broiler chickens fed conventional or antibiotic-free vegetable-based diets. J Appl Microbiol. 2007;102:1138-49. https://doi.org/10.1111/j.1365-2672.2006.03153.x.

27. Tillman GE, Haas GJ, Wise MG, Oakley B, Smith MA, Siragusa GR. Chicken intestine microbiota following the administration of lupulone, a hop-based antimicrobial. FEMS Microbiol Ecol. 2011;77:395-403. https://doi.org/10.1111/ j.1574-6941.2011.01119.x.

28. Wei S, Morrison M, Yu Z. Bacterial census of poultry intestinal microbiome. Poult Sci. 2013;92:671-83. https://doi.org/10.3382/ps.2012-02822.

29. Oakley BB, Lillehoj HS, Kogut MH, Kim WK, Maurer JJ, Pedroso A, et al. The chicken gastrointestinal microbiome. FEMS Microbiol Lett. 2014;360:100-12. https://doi.org/10.1111/1574-6968.12608.

30. Shin NR, Whon TW, Bae JW. Proteobacteria: microbial signature of dysbiosis in gut microbiota. Trends Biotechnol. 2015;33:496-503. https://doi.org/10. 1016/j.tibtech.2015.06.011.

31. Mukhopadhya I, Hansen R, El-Omar EM, Hold GL. IBD-what role do Proteobacteria play? Nat Rev Gastroenterol Hepatol. 2012;9:219-30. https:// doi.org/10.1038/nrgastro.2012.14. 
32. Kers JG, Velkers FC, Fischer EAJ, Hermes GDA, Stegeman JA, Smidt H. Host and environmental factors affecting the intestinal microbiota in chickens. Front Microbiol. 2018;9:235. https://doi.org/10.3389/fmicb.2018.00235.

33. Gong J, Si W, Forster RJ, Huang R, Yu H, Yin Y, et al. 16S rRNA gene-based analysis of mucosa-associated bacterial community and phylogeny in the chicken gastrointestinal tracts: from crops to ceca. FEMS Microbiol Ecol. 2007:59:147-57. https://doi.org/10.1111/j.1574-6941.2006.00193.x.

34. Danzeisen JL, Kim HB, Isaacson RE, Tu ZJ, Johnson TJ. Modulations of the chicken cecal microbiome and metagenome in response to anticoccidial and growth promoter treatment. PLoS One. 2011;6:e27949. https://doi.org/ 10.1371/journal.pone.0027949.

35. Stanley D, Denman SE, Hughes RJ, Geier MS, Crowley TM, Chen H, et al. Intestinal microbiota associated with differential feed conversion efficiency in chickens. Appl Microbiol Biotechnol. 2012;96:1361-9. https://doi.org/10. 1007/s00253-011-3847-5.

36. Costa MC, Bessegatto JA, Alfieri AA, Weese JS, Filho JA, Oba A. Different antibiotic growth promoters induce specific changes in the cecal microbiota membership of broiler chicken. PLoS One. 2017;12:e0171642. https://doi.org/10.1371/journal.pone.0171642.

37. Borrelli L, Coretti L, Dipineto L, Bovera F, Menna F, Chiariotti L, et al. Insectbased diet, a promising nutritional source, modulates gut microbiota composition and SCFAs production in laying hens. Sci Rep. 2017;7:16269. https://doi.org/10.1038/s41598-017-16560-6.

38. Xue Z, Zhang W, Wang L, Hou R, Zhang M, Fei L, et al. The bambooeating giant panda harbors a carnivore-like gut microbiota, with excessive seasonal variations. MBio. 2015;6:e00022-15. https://doi.org/10. 1128/mBio.00022-15.

39. Kuhn I, Katouli M, Lund A, Wallgren P, Mollby R. Phenotype diversity and stability of intestinal coliform flora in piglets during the first three months of age. Microb Ecol Health Dis. 1993;6:101-7. https://doi.org/10.3109/ 08910609309141313.

40. Oliphant K, Allen-Vercoe E. Macronutrient metabolism by the human gut microbiome: major fermentation by-products and their impact on host health. Microbiome. 2019;7:91. https://doi.org/10.1186/s40168-019-0704-8.

41. Li Z, Wang W, Liu D, Guo Y. Effects of Lactobacillus acidophilus on gut microbiota composition in broilers challenged with Clostridium perfringens. PLoS One. 2017;12:e0188634. https://doi.org/10.1371/journal.pone.0188634.

42. Jung T-H, Park JH, Jeon W-M, Han K-S. Butyrate modulates bacterial adherence on LS174T human colorectal cells by stimulating mucin secretion and MAPK signaling pathway. Nutr Res Pract. 2015;9:343-9. https://doi.org/10.4162/nrp.2015.9.4.343.

43. Peng L, Li Z-R, Green RS, Holzman IR, Lin J. Butyrate enhances the intestinal barrier by facilitating tight junction assembly via activation of amp-activated protein kinase in caco-2 cell monolayers. J Nutr. 2009;139:1619-25. https:// doi.org/10.3945/jn.109.104638.

44. Rinttila T, Apajalahti J. Intestinal microbiota and metabolites-implications for broiler chicken health and performance. J Appl Poult Res. 2013;22:647-58. https://doi.org/10.3382/japr.2013-00742.

45. Furusawa Y, Obata Y, Fukuda S, Endo TA, Nakato G, Takahashi D, et al. Commensal microbe-derived butyrate induces the differentiation of colonic regulatory T cells. Nature. 2013;504:446-50. https://doi.org/10.1038/nature12721.

46. Louis P, Flint HJ. Diversity, metabolism and microbial ecology of butyrateproducing bacteria from the human large intestine. FEMS Microbiol Lett 2009;294:1-8. https://doi.org/10.1111/j.1574-6968.2009.01514.x.

47. Liu C, Finegold SM, Song Y, Lawson PA. Reclassification of Clostridium coccoides, Ruminococcus hansenii, Ruminococcus hydrogenotrophicus, Ruminococcus luti, Ruminococcus productus and Ruminococcus schinkii as Blautia coccoides gen. nov., comb. nov., Blautia hansenii comb. nov., Blautia hydrogenotrophica comb. nov., Blautia luti comb. nov., Blautia producta comb. nov., Blautia schinkii comb. nov. and description of Blautia wexlerae sp. nov., isolated from human faeces. Int J Syst Evol Microbiol. 2008;58: 1896-902. https://doi.org/10.1099/ijs.0.65208-0.

48. Sunkara LT, Jiang W, Zhang G. Modulation of antimicrobial host defense peptide gene expression by free fatty acids. PLoS One. 2012;7:e49558. https://doi.org/10.1371/journal.pone.0049558.

49. Rehman HU, Vahjen W, Awad WA, Zentek J. Indigenous bacteria and bacterial metabolic products in the gastrointestinal tract of broiler chickens. Arch Anim Nutr. 2007;61:319-35. https:/doi.org/10.1080/17450390701556817.

50. Pryde SE, Duncan SH, Hold GL, Stewart CS, Flint HJ. The microbiology of butyrate formation in the human colon. FEMS Microbiol Lett. 2002;217:133-9. https://doi.org/10.1111/j.1574-6968.2002.tb11467.x.
51. Van Tassell ML, Miller MJ. Lactobacillus adhesion to mucus. Nutrients. 2011; 3:613-36. https://doi.org/10.3390/nu3050613.

52. Ren CC, Zhang QX, de Haan BJ, Zhang H, Faas MM, de Vos P. Identification of TLR2/TLR6 signalling lactic acid bacteria for supporting immune regulation. Sci Rep. 2016;6:34561. https://doi.org/10.1038/srep34561.

53. Elferink S, Krooneman J, Gottschal JC, Spoelstra SF, Faber F, Driehuis F. Anaerobic conversion of lactic acid to acetic acid and 1,2-propanediol by Lactobacillus buchneri. Appl Environ Microbiol. 2001;67:125-32. https://doi. org/10.1128/AEM.67.1.125-132.2001.

54. Duncan $\mathrm{SH}$, Louis $\mathrm{P}$, Flint HJ. Lactate-utilizing bacteria, isolated from human feces, that produce butyrate as a major fermentation product. Appl Environ Microbiol. 2004;70:5810-7. https://doi.org/10.1128/AEM.70.10.5810-5817.2004.

55. Bjerrum L, Engberg RM, Leser TD, Jensen BB, Finster K, Pedersen K. Microbial community composition of the ileum and cecum of broiler chickens as revealed by molecular and culture-based techniques. Poult Sci. 2006;85: 1151-64. https://doi.org/10.1093/ps/85.7.1151.

56. Chang $\mathrm{CL}$, Chung CY, Kuo CH, Kuo TF, Yang CW, Yang WC. Beneficial effect of bidens pilosa on body weight gain, food conversion ratio, gut bacteria and coccidiosis in chickens. PLoS One. 2016;11:e0146141. https://doi.org/10. 1371/journal.pone.0146141.

57. Wrigley DM. Inhibition of Clostridium perfringens sporulation by Bacteroides fragilis and short-chain fatty acids. Anaerobe. 2004;10:295-300. https://doi. org/10.1016/j.anaerobe.2004.05.006

58. Scott KP, Martin JC, Chassard C, Clerget M, Potrykus J, Campbell G, et al. Substrate-driven gene expression in Roseburia inulinivorans: importance of inducible enzymes in the utilization of inulin and starch. Proc Natl Acad Sci U S A. 2011;108:4672-9. https://doi.org/10.1073/pnas.1000091107.

59. Stanley J, Linton D, Burnens AP, Dewhirst FE, On SL, Porter A, et al. Helicobacter pullorum sp nov-genotype and phenotype of a new species isolated from poultry and from human patients with gastroenteritis. Microbiol. 1994;140:3441-9. https://doi.org/10.1099/13500872-140-12-3441.

60. Forder RE, Howarth GS, Tivey DR, Hughes RJ. Bacterial modulation of small intestinal goblet cells and mucin composition during early posthatch development of poultry. Poult Sci. 2007;86:2396-403. https://doi.org/10. 3382/ps.2007-00222.

61. Dean-Nystrom EA, Samuel JE. Age-related resistance to 987P fimbriamediated colonization correlates with specific glycolipid receptors in intestinal mucus in swine. Infect Immun. 1994;62:4789-94 Manca doi.

62. Corfield AP, Wagner SA, Clamp JR, Kriaris MS, Hoskins LC. Mucin degradation in the human colon: production of sialidase, sialate O-acetylesterase, $\mathrm{N}$ acetylneuraminate lyase, arylesterase, and glycosulfatase activities by strains of fecal bacteria. Infect Immun. 1992;60:3971-8 Manca doi.

63. Turck D, Feste A, Lifschitz C. Age and diet affect the composition of porcine colonic mucins. Pediatr Res. 1993;33:564-7. https://doi.org/10.1203/ 00006450-199306000-00005.

64. Uni Z, Smirnov A, Sklan D. Pre- and posthatch development of goblet cells in the broiler small intestine: effect of delayed access to feed. Poult Sci. 2003:82:320-7. https://doi.org/10.1093/ps/82.2.320.

65. Uni Z, Platin R, Sklan D. Cell proliferation in chicken intestinal epithelium occurs both in the crypts and along the villus. J Comp Physiol B. 1998;168: 241-7. https://doi.org/10.1007/s003600050142.

66. Wang JX, Peng KM. Developmental morphology of the small intestine of African ostrich chicks. Poult Sci. 2008;87:2629-35. https://doi.org/10.3382/ps. 2008-00163.

Ready to submit your research? Choose BMC and benefit from:

- fast, convenient online submission

- thorough peer review by experienced researchers in your field

- rapid publication on acceptance

- support for research data, including large and complex data types

- gold Open Access which fosters wider collaboration and increased citations

- maximum visibility for your research: over $100 \mathrm{M}$ website views per year

At $\mathrm{BMC}$, research is always in progress.

Learn more biomedcentral.com/submission 